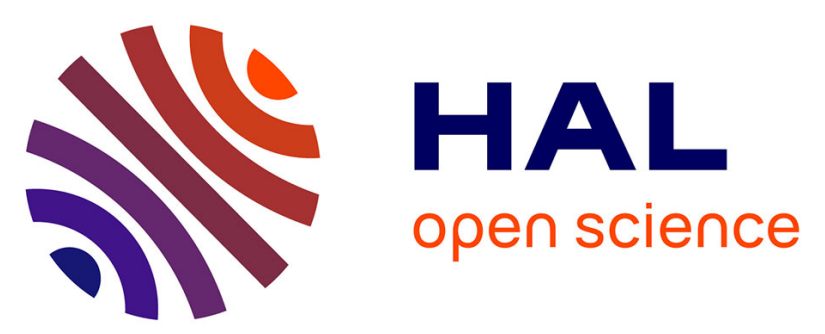

\title{
Full-Scale Discrete Event Simulation of an Automated Modular Conveyor System for Warehouse Logistics
}

\author{
Alireza Ashrafian, Ole-Gunnar Pettersen, Kristian N. Kuntze, Jacob Franke, \\ Erlend Alfnes, Knut F. Henriksen, Jakob Spone
}

\section{To cite this version:}

Alireza Ashrafian, Ole-Gunnar Pettersen, Kristian N. Kuntze, Jacob Franke, Erlend Alfnes, et al.. FullScale Discrete Event Simulation of an Automated Modular Conveyor System for Warehouse Logistics. IFIP International Conference on Advances in Production Management Systems (APMS), Sep 2019, Austin, TX, United States. pp.35-42, 10.1007/978-3-030-29996-5_4 . hal-02460491

\section{HAL Id: hal-02460491 \\ https://hal.inria.fr/hal-02460491}

Submitted on 30 Jan 2020

HAL is a multi-disciplinary open access archive for the deposit and dissemination of scientific research documents, whether they are published or not. The documents may come from teaching and research institutions in France or abroad, or from public or private research centers.
L'archive ouverte pluridisciplinaire HAL, est destinée au dépôt et à la diffusion de documents scientifiques de niveau recherche, publiés ou non, émanant des établissements d'enseignement et de recherche français ou étrangers, des laboratoires publics ou privés. 


\title{
Full-Scale Discrete Event Simulation of an Automated Modular Conveyor System for Warehouse Logistics
}

\author{
Alireza Ashrafian ${ }^{1}$, Ole-Gunnar Pettersen ${ }^{1}$, Kristian N. Kuntze ${ }^{1}$, Jacob Franke ${ }^{1}$, \\ Erlend Alfnes ${ }^{1}$, Knut F. Henriksen ${ }^{2}$, Jakob Spone ${ }^{3}$ \\ ${ }^{1}$ Norwegian University of Science and Technology, Trondheim 7491, Norway \\ ${ }^{2}$ Swisslog, Oslo 0581, Norway \\ ${ }^{3}$ ASKO, Oslo 0950, Norway \\ alireza.ashrafianentnu.no
}

\begin{abstract}
This paper presents the use of advanced simulation modeling to optimize the operation of a fully automated modular conveyor system in a large-scale warehouse. At its peak capacity, the smooth flow of material in the system was greatly impaired due to the appearance of bottlenecks. A full-scale 3D discrete event simulation (DES) model of the system was built and time-dependent statistical models were carefully designed and implemented in the model in order to capture the randomness and complex dynamics of the operation. The model was verified and validated, and several scenarios have been analyzed. The paper demonstrates a practical example of how data-driven simulation modeling provided a cost-effective solution to enhance efficiency. The paper highlights the crucial aspects that must be taken into account in the modeling of the system in order to create a reliable standalone decision support system. Moreover, the paper highlights the identified key steps that are yet to be taken from dynamical modelling towards a Digital Twin.
\end{abstract}

Keywords: Discrete Event Simulation, Discrete Event Logistics System, Warehouse Logistics, Distribution Center Automation, Material Handling, Modular Conveyor Systems, Decision Support System.

\section{Introduction}

Automated modular conveyor systems are widely used in central warehouses and material distribution centers to achieve a high degree of throughput and smooth material flow while remaining flexible and efficient [4]. Achieving high efficiency, however, is not always problem free. The higher capacity and increased complexity of interactions in smart material handling systems also require advanced analysis to avoid design mistakes and to ensure efficient operation. Factors such as varying demand and a high degree of randomness, this poses significant challenges in achieving desired ef- 
ficiencies and maintaining smooth material flows. Verifying that the system design adheres to operational requirements under constantly varying conditions is often beyond the capability of conventional analysis tools [3].

In the past few decades, computer simulation has become one of the most effective decision support tools used in logistic systems. 3D transient computer simulations have provided reliable solutions to identify and avoid costly design and operational mistakes. Among them, discrete event simulation (DES) [5] has become an indispensable tool for understanding the complex dynamics in logistics and supply chain systems [2,9].

Moreover, and within the paradigm of Industry 4.0 digital transformation, computer simulations integrated with virtual reality systems and operation data, have become the basis of the digital twin technology [6,7]. According to Stark et al. [8], "A Digital Twin is the digital representation of a unique asset (product, machine, service, product service system or other intangible asset), that alters its properties, condition and behavior by means of models, information and data". A Digital Twin is a digital abstraction of a system that is fed with live, high quality, data. The vision of a digital twin for smart warehouse systems is, therefore, to create a mirror of processes incorporating all related information within the warehouse system. The twin can be used to monitor processes in real time and continuously fine tune all operations in the warehouse in order to optimize the flow, to find bottlenecks, etc. In short, if such a digital twin is realized, it allows better understand end-to-end processes, find bottlenecks and improve performance beyond all expectations. The success of digital twins is, therefore, greatly dependent on, i.a., the performance of the underlying simulation model in capturing the key characteristics and complex dynamics of the system.

The purpose of this paper is to demonstrate how a full scale DES model can support the design and optimization of smart warehouse systems. The paper also clarifies what are the crucial aspects that must be taken into account in full detail in the model in order to create a reliable standalone decision support system. Moreover, the paper highlights the key steps that are yet to be taken to make the dynamical model a real Digital Twin that can be used as an effective tool for decision-making and optimization.

\section{The automated modular conveyor system under study}

\subsection{The geometry and operation}

The system under study is an automated modular conveyor system for handling totes, trays and cartons [4] that has a complex interconnected geometry (Fig. 1). The system comprises of five major parts: the "supplier", the mini-load storage systems, the "highway", 5 sub-loops, and 21 pickup stations. The supplier feeds the system with totes that are stacked with products from a pallet. The highway is the central conveyor that runs through the system and makes it possible that totes fed by the supplier can travel between the sub-loops. Typically, a tote that comes from the supplier via the highway to a sub-loop will end up at a mini-load station for storage. Mini-loads are vertical storage units that store loaded totes and trays for later use. Each mini-load system is connected to a sub-loop in the conveyor system and has 5 input and output ports. Alternatively, if a tote enters the system through a mini-load, it will usually go to one of the four pickup 
stations connected to the associated sub-loop. These pick up stations hold a tote for a statistically certain amount of time before it is released to its next destination, e.g., another pick up station.

All routing operations are fully automated by scanner and barcodes. In front of each decision point, the control system sends a totes barcode to a bin destination manager (BDM), which immediately returns a command telling the control system if the tote should proceed straight ahead or change direction. The BDM is a part of the central warehouse management system (WMS). Each module has a constraint on capacity, i.e., it can contain a limited number of totes in order to function properly. Congestion occurs if this limit is exceeded and, consequently, the smooth function of the system will be impaired. To prohibit congestion, all of the entrances to and the exits from the areas are equipped with optical counters that control the number of totes in that section. The system automatically blocks further incoming of totes if the maximum capacity is reached.

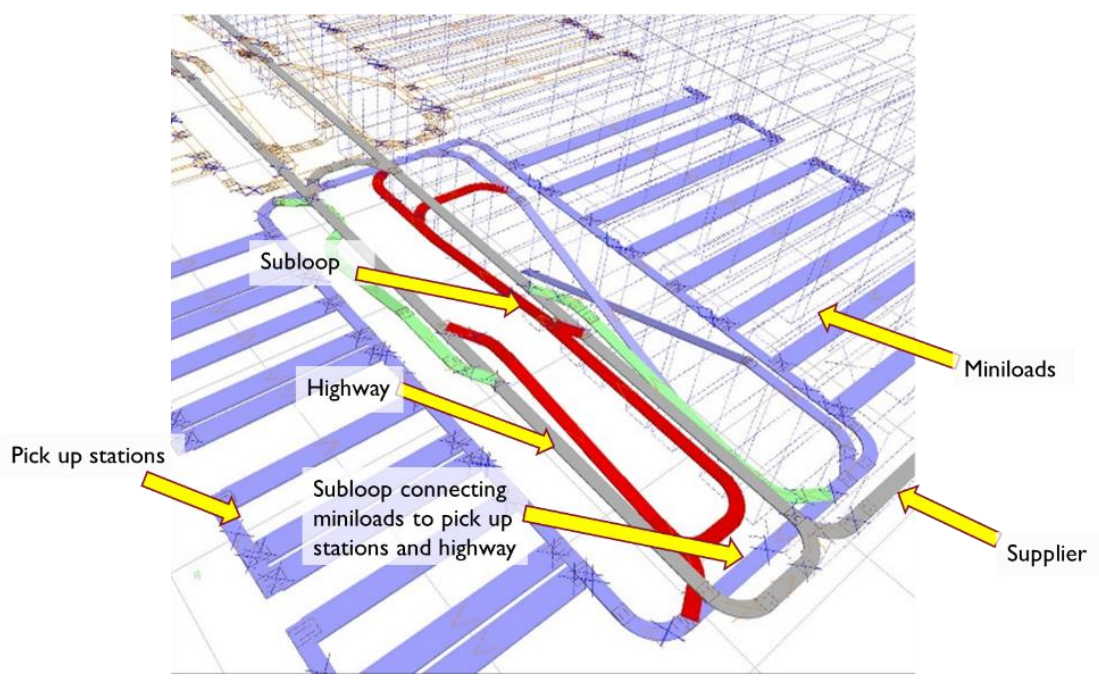

Fig. 1. The geometry of the modular conveyor system under study (Courtesy of ASKO and Swisslog).

\section{$2.2 \quad$ Operational challenges}

The system is designed such that the bottleneck in the system always resides at the pickup stations. This is to ensure the continuous flow of totes in other parts of the system and, to ensure that totes are always available for the operators at pick up stations. The operators have a relatively high operational cost, and they should never be idle.

At times, during periods of increased strain on the system, the highway often becomes the bottleneck and the flow of totes is disrupted. Several observations from operating the system suggested that the bottleneck appeared on the highway due to its capacity limitation as well as the way the supplier feeds the highway which takes a lot of its capacity. The current simulation modeling and analysis was therefore aimed at 
finding a new way of feeding the totes from the supplier to the system and, support the corresponding design changes with data-driven analysis.

\section{The full-scale dynamical model}

The simulation modeling and analysis software FlexSim ${ }^{\circledR}[1]$ has been used in order to perform a full scale DES model of the automated modular conveyor system under study. The model geometry is based on the 3D CAD data and is identical to the real system in operation (Fig. 2).

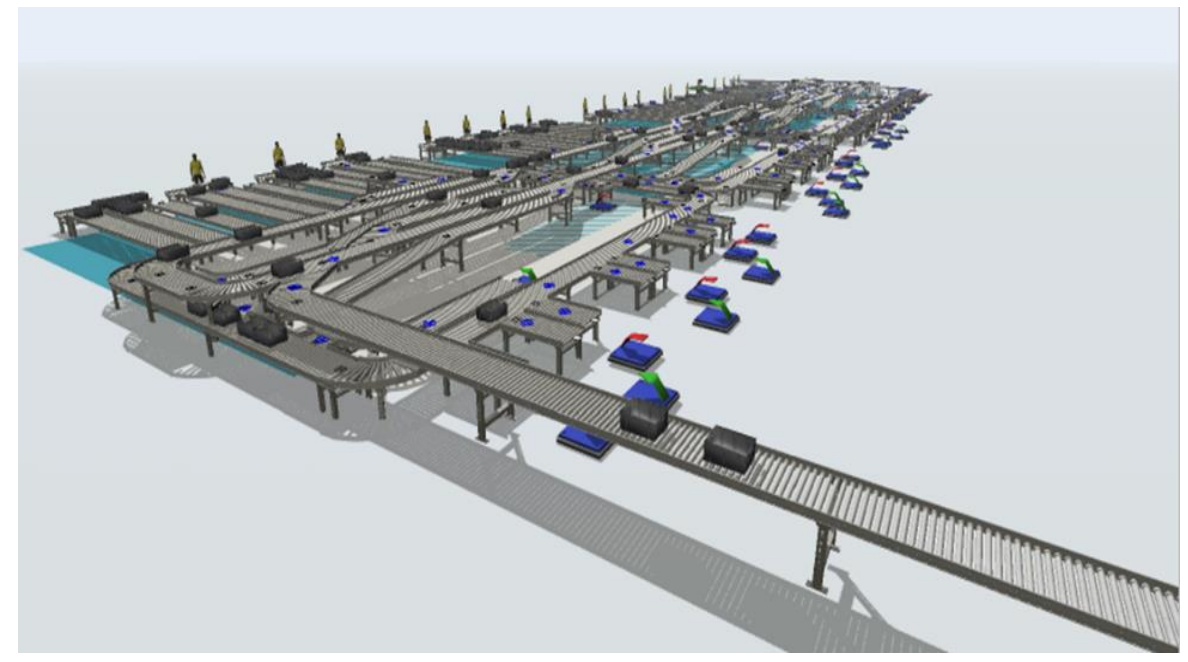

Fig. 2. The full-scale computer simulation model for the installed modular conveyor system.

\subsection{The model logic}

The operational logic of the system has several important aspects that are carefully implemented in the model. There are certain variables tagged to each individual tote that contains information about its current state and destination, as well as an individual name that is used for statistics collection and, later on, for model verification. These variables come into play at every crossroad where decision points are located. Right before every crossroad, where a tote is faced with several different paths to continue on, there is a decision point that contains a code attributed to the variables in each tote to decide whether to continue on to one conveyor or another. A decision point may also change the variables containing information about the totes destination, e.g., by choosing what pickup station to travel to right after entering a sub-loop.

As mentioned before, when a tote has arrived at a pickup station, it will be held at that station for a certain amount of time. This stay time varies statistically depending on the work of operator at that station. The pickup station also changes the information of the tote upon its release. For example, when the pickup station labels a tote as 
"empty", then the decision points are forced to direct the tote to the fastest available route to exit the system. The final part of the logic of the operation deals with the function of suppliers which provide the model with totes based on statistical distributions that change over time (see \$3.2). They also create and set the value of the variables in the tote. The value of these variables is also determined by time-dependent statistical distributions. The associated operational logic explained above is programmed in each corresponding individual element of the model using the script language provided by the FlexSim ${ }^{\circledR}$ software [1].

\subsection{Simulation input and statistical modelling}

Various time-dependent statistical data from the operation is integrated with the model. The data was collected from the system over one week of operation amounting to approximately 500,000 totes. The data included the number of totes arriving at the system per hour, and the number of totes being processed by the system per hour.

Moreover, statistical analysis was performed in order to model the statistical distributions [5] for the various number of input variables such as manual feeding rate to the supplier, feeding rates from the mini-loads, processing time at pick up stations, and the decision points that distribute the totes throughout the system. Hourly based operational data indicated a high degree of dynamics in the variations associated with these statistical distributions. A considerable effort was made in order to correctly model these variations and program it in the DES model.

\subsection{Model verification and validation}

Several verifications of the model were performed under controlled conditions in order to ensure the consistency of the model and its internal logic. Validation of the model including various statistical models was also performed by comparing the simulation results with operational data for several variables. To ensure the consistency of the model in terms continuity between all geometrical areas, the totes resident time in the system were calculated and it was verified that no tote remains in the system for a large amount of time. The statistical correctness of decision points in sending totes to various destinations was validated by comparing the simulation results for the cumulative number of totes ended up at various sub-loops with the operational data after several hours of operation (Fig. 3). 


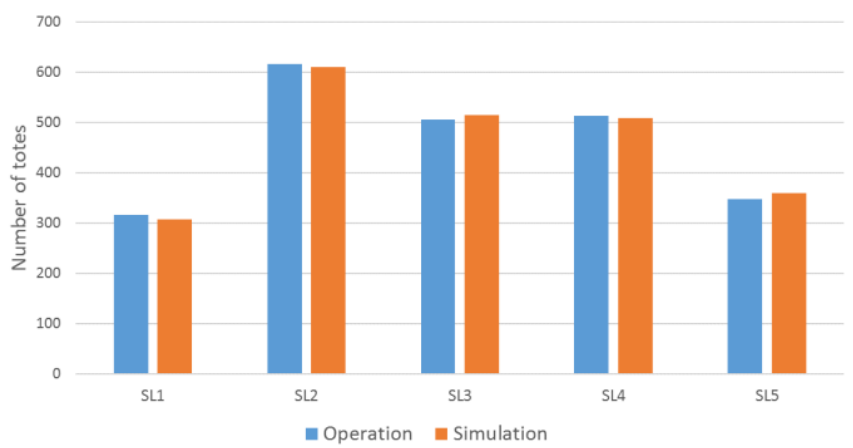

Fig. 3. Cumulative number of totes sent to various sub-loops (SLs) by the decision points.

\section{$4 \quad$ Results and the improved design}

Various simulations were performed considering multiple scenarios for the design modifications in the system. The resulting scenario that had the most effect on removing the bottleneck from the highway and therefore improving the smooth material flow in the system is presented here. The reference case is the one identical to the conditions in the original system. The analysis was based on various key performances in the system, e.g., average content in the highway and the sub-loops, idle time for the operators at pick up stations, and passages through certain decision points along the highway. These give clear indications of how the implementation of the suggested design modification would affect the system.

The proposed new design (Fig. 4) contains a new loop where the newly stacked totes would go alongside the highway and further on to the allotted sub-loops, and finally, the mini-load for storage. The basic idea was to ensure the increased availability of totes for the pickup station, while reducing the average number of totes on the highway, and hence, avoiding congestion. It should be noted that the time-dependent statistical data for the number of totes arriving at the system as well as the statistical distributions for e.g., manual feeding to the system remains unchanged and equally applicable to the system with new design as well. The average content in the entire system dropped by about $8 \%$ percent. Results for the highway and each of the sub-loops are shown in Fig. 5 . Note that the number of totes processed in each sub-loop has remained almost unaffected. The number of totes passing through the decision points on the highway which dropped by a staggering $28 \%$ (not shown here) which clearly indicates a significant reduction in the traffic on the highway. Another advantage of the design is that if one of the sub-loops are full, the tote could loop around instead of congesting the highway. Moreover, an increase in the availability of the totes to the operators without consuming further from the available area in the facility was obtained. 


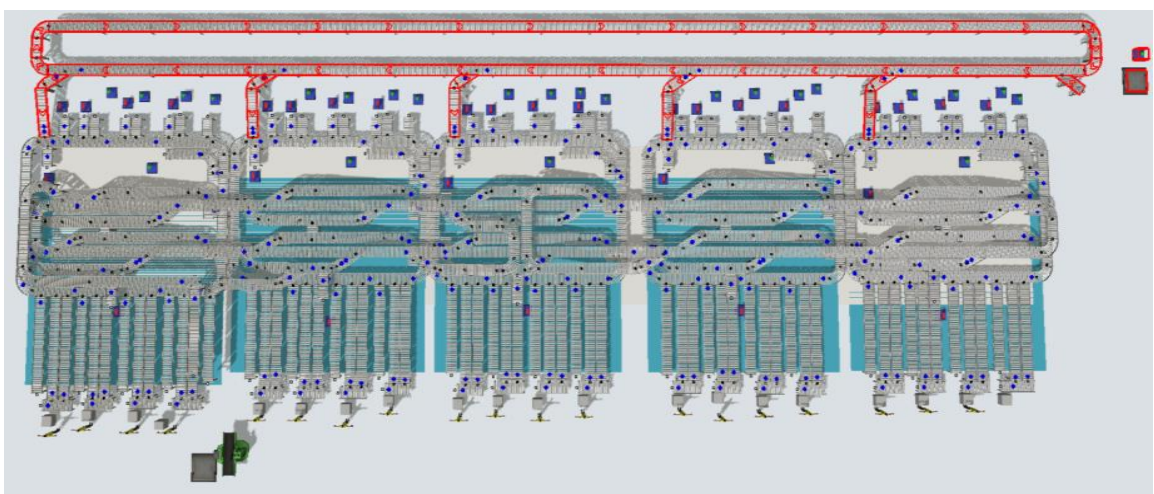

Fig. 4. The proposed new design including a new conveyor loop marked in red.

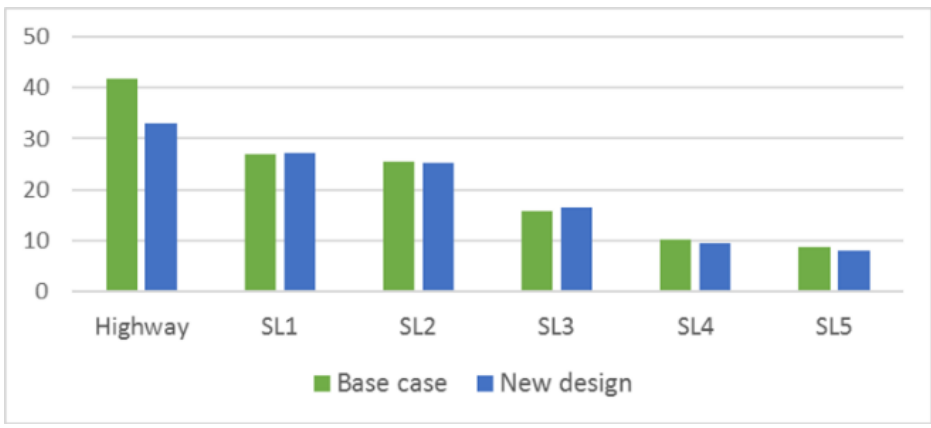

Fig. 5. Average number of totes per hour on the highway and sub-loops.

Fig. 6 shows a heat map of the average tote content on the highway. The reduction in the number of totes on the highway is evident, especially, in the area closest to the entry from the supplier (the right end of the figure). The analyses point out the apparent reduction of strain put on the system and a more uniform distribution of totes along the whole highway conveyor.

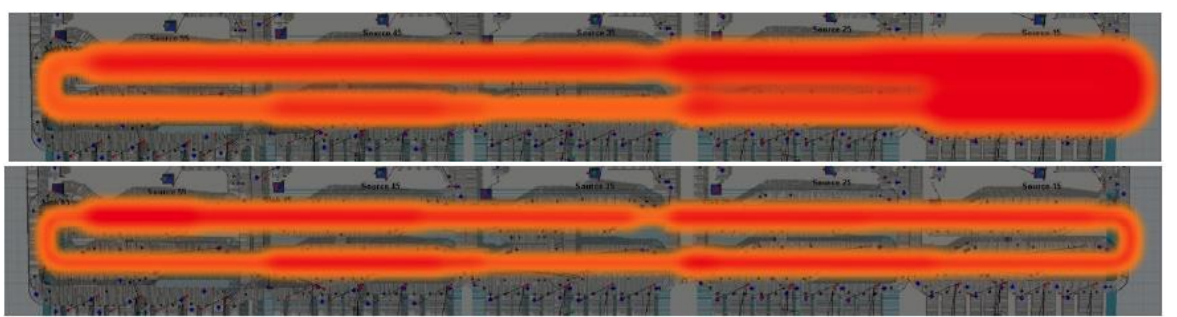

Fig. 6. Heat maps indicating the average number of totes on the highway conveyor. Top: base case, bottom: the new design. 


\section{Conclusions}

Highly automated logistics systems incorporate enhanced capacity and efficiency as well as a higher degree of complexity in system dynamics. To this end, advanced computer simulation models are of great value to create near-reality standalone models for complex discrete event logistics system (DELS). The model presented in this study has captured the complex geometry, the variety of system interactions, the system logic, and the key stochastic dynamics that took place under the real operation. It was demonstrated that the current model could be used as a reliable standalone decision support system for operation and design change. Throughout the course of this study, some requirements were also identified in order to make conventional simulation modeling an effective tool for Digital Twins. Firstly, connectivity and integration with operational or enterprise resource planning database must be realized. Secondly, and as demonstrated in this study, the modelled system for Digital Twin application must include high level of details and low level of abstraction in all the aspects including geometry, stochastic and logic. Ultimately, the construction of models, the process of optimization through design modifications, development of model versions, and the construction of various scenarios should be automated to the highest degree in order to achieve efficiency without manual interventions. Moreover, the need for functionality was of significant importance in the course of conducting the current study. Manual modifications of the simulation model can be time consuming, especially if a large set of variations of the model needs to be built.

\section{References}

1. Beaverstock, M., Greenwood, A. G., Lavery, E. \& Nordgren, B. (2017). “Applied Simulation: Modeling and Analysis Using FlexSim", FlexSim Software Products Inc., Orem, USA.

2. Brailsford, S., Dangerfield, B. \& Churilov, L. red. (2014). "Discrete-Event Simulation and System Dynamics for Management Decision Making”, John Wiley \& Sons, Ltd.

3. Jia, Y., Jiang, P. F. (2017). "The Application of Simulation Technology in Distribution Center", Applied Mechanics and Materials; Zurich, Vol. 865: Pages 675-680.

4. "QuickMove: Flexible, modular conveyor system for small loads", Swisslog Holding Ltd., 2018, https://www.swisslog.com/quickmove, accessed April 2018.

5. Law, A. M. (2015). Simulation Modeling and Analysis. $5^{\text {th }}$ ed., McGraw-Hill.

6. Rodic, B. (2017). Industry 4.0 and the New Simulation Modelling Paradigm, Organizacija, Volume 50, Number 3, 193

7. Rosen, R., von Wichert, G., Lo, G., \& Bettenhausen, K. D. (2015). About The Importance of Autonomy and Digital Twins for the Future of Manufacturing. IFAC-PapersOnLine, 483, Pages 567-572.

8. Stark, R., Kind, S., \& Neumeyer, S. (2017). Innovations in digital modelling for next generation manufacturing system design. CIRP Annals - Manufacturing Technology, Volume 66, Issue 1, Pages 169-172

9. Tako, A., A., Stewart, R. (2012). "The application of discrete event simulation and system dynamics in the logistics and supply chain context", Decision Support Systems, Volume 52, Issue 4, March 2012, Pages 802-815. 